\title{
Synthesis and Characterization of AgPd/Pt Multiply Twinned Structure Nanoparticles by Using Cs-Corrected STEM
}

Subarna Khanal, J. J. Velazquez-Salazar, N. Bhattarai, D. Bahena, G. Casillas, A. Ponce, and M. Jose-Yacaman

Department of Physics and Astronomy, University of Texas at San Antonio, One UTSA Circle, San Antonio, Texas, 78249

The synthesis of noble metals (e.g. Ag, Pd, Pt, Au etc.) nanostructure with controlled atomic distributions have attracted significant interest due to their size-tunable properties depending up on the variation of composition and structure. Recently, various researchers are fascinated in multimetallic nanoparticles, because of their diverse potential applications different from monometallic constituents. Controlling the morphology of nanostructures can provide a great opportunity to stimulate their possible applications such as catalytic, electronic, and optical properties and to enhance their reactivity, selectivity and stability [1,2]. The fabrication of $\mathrm{AgPd} / \mathrm{Pt}$ core-shell trimetallic nanoparticles with multiply twinned structures, possessing the icosahedra morphology with a high density of compressed twins and high fraction of Pt atoms on the (111) surfaces are expected to be the most active catalysts for their many applications in fuel cells $[3,4]$.

In order to characterization of the as prepared $\mathrm{AgPd} / \mathrm{Pt}$ core-shell trimetallic nanoparticles, the transmission electron microscopy (TEM) was employed to observe the morphology of the sample. The scanning transmission electron microscopy (STEM) images were recorded in probe Cs-corrected JEOL JEM-ARM 200F operated at $200 \mathrm{kV}$. HAADF STEM images were obtained with a convergence angle of $26 \mathrm{mrad}$ and the collection semi-angles from 50 to $180 \mathrm{mrad}$. These variations in semi-angles satisfy the conditions set forth for the detectors to eliminate contributions from unscattered and low-angle scattered electron beams. The probe size used was about $0.09 \mathrm{~nm}$ with the probe current of $22 \mathrm{pA}$. In addition, bright field (BF) STEM images were recorded by using a collection semi-angle of $11 \mathrm{mrad}$. Electron dispersive $\mathrm{x}$-ray spectroscopy (EDS) spectra were obtained using a probe size of $0.13 \mathrm{~nm}$ with the probe current $86 \mathrm{pA}$.

In this present work, we have successfully synthesized $\mathrm{AgPd} / \mathrm{Pt}$ core-shell multiply twinned nanoparticles with icosahedral morphology. The Cs-corrected scanning transmission electron microscopy (Cs-corrected STEM) in combination with high angle annular dark field (HAADF), bright field (BF) and energy dispersive X-ray analysis (EDS) detectors allowed us to probe the structural information at the atomic level of these nanoparticles revealing the core, and the shell regions such that the core is AgPd alloy and the shell is Pt. Moreover, the line profile of the EDS analysis reveals that the elemental composition of these nanostructures and it confirms the structures are core-shell, which is expected to be the most active catalysts. 


\section{References}

[1] G. Wang, M. A. Van Hove, P. N. Ross and M. I. Baskes, Progress in Surface Science, 2005, $79,28$.

[2] S. Khanal, G. Casillas, J. J. Velazquez-Salazar, A. Ponce and M. Jose-Yacaman, The Journal of Physical Chemistry C, 2012, 116, 23596.

[3] C. Li, R. Sato, M. Kanehara, H. Zeng, Y. Bando and T. Teranishi, Angewandte Chemie, 2009, 121, 7015.

[4] J. Yang, J. Yang and J. Y. Ying, ACS Nano, 2012, 6, 9373.

[5] Acknowledgments: The authors would like to acknowledge NSF grants DMR-1103730, "Alloys at the Nanoscale: The Case of Nanoparticles Second Phase" and PREM: NSF PREM Grant \# DMR 0934218
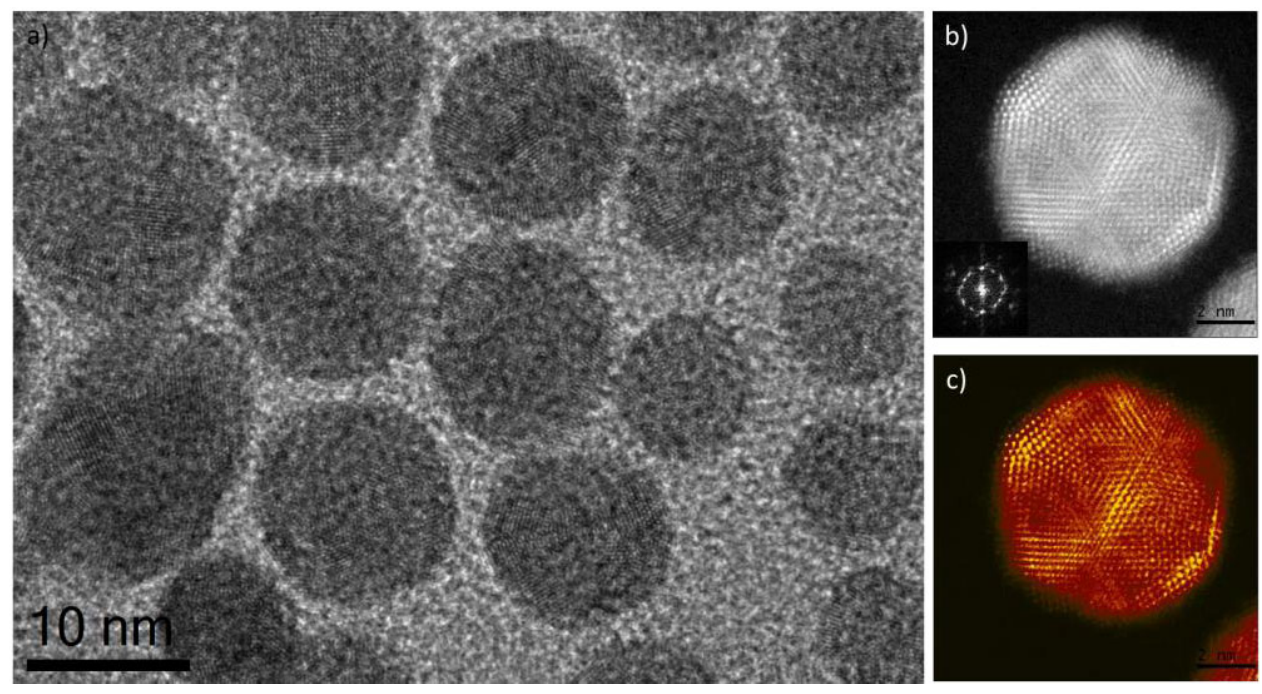

Figure 1. (a) HRTEM image of the icosahedral $\mathrm{AgPd} / \mathrm{Pt}$ trimetallic core-shell nanoparticles, (b) The atomic resolution HAADF-STEM image of the icosahedral shape (inset shows FFT pattern), and (c) Color image shows the different intensity of different elements.
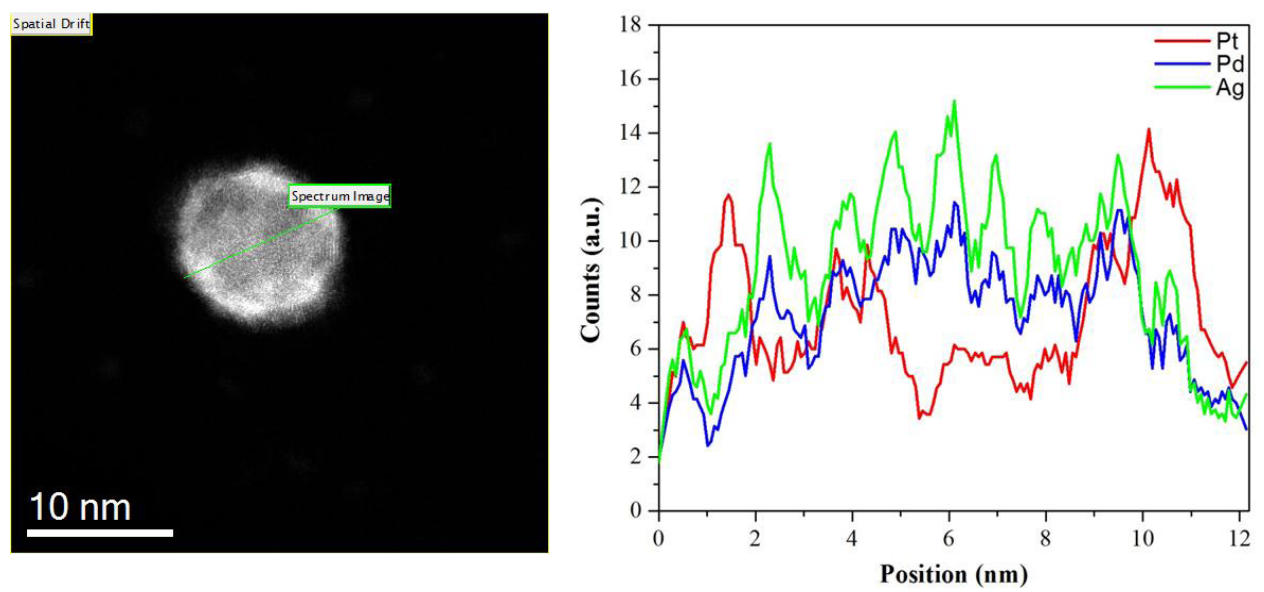

Figure 2. HAADF-STEM image and Ag, Pd and Pt elemental profiles along the green line (left figure) across the icosahedral multiple twined particles. 